\title{
Fatty liver and the metabolic syndrome-fructose at fault?
}

New research demonstrates the crucial role of endogenous fructose production and metabolism in the development of fatty liver and the metabolic syndrome. Reporting in Nature Communications, Miguel Lanaspa and colleagues show that the metabolic conversion of glucose (consumed in drinking water) to endogenous fructose in the liver is a key step in the development of glucose-induced obesity and fatty liver in mice.

Rates of obesity and the metabolic syndrome are increasing worldwide at a worrying pace. Although excessive carbohydrate intake has been linked to the development of obesity and fatty liver, the underlying mechanisms are unclear.

Lanaspa et al. monitored the effects of high levels of glucose (10\% glucose in drinking water for 10 weeks) on mice unable to metabolise fructose; that is, fructokinase-knockout (termed KHK-A/C KO) mice. In comparison with wild-type mice, KHK-A/C KO mice had markedly less weight gain,

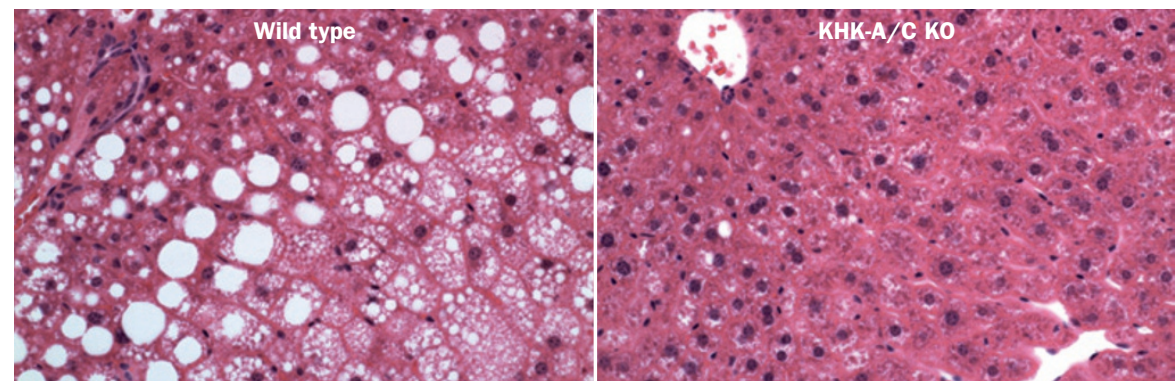

Macrosteatosis in wild-type but not fructokinase-deficient mice. Permission obtained from Nature Publishing Group $\odot$ Lanaspa, M. A. et al. Nat. Commun. 4, 2434 (2013).

visceral obesity and hyperinsulinaemia. Strikingly, KHK-A/C KO mice did not develop glucose-induced fatty livers; upon histological examination, steatosis was visible only in the livers of wild-type mice. Moreover, activation of aldose reductase and the polyol pathway in the liver was determined to be critical for the endogenous conversion of glucose into fructose and the resultant negative effects observed in wild-type mice on a highglucose diet. Importantly, mice deficient in aldose reductase had reduced body and fat weight, and reduced levels of fatty liver.
Lanaspa states that their findings imply that even foods with a low glycaemic index (such as fructose) can contribute to the development of fatty liver and the metabolic syndrome, and those with a high glycaemic index contribute via the conversion of glucose to fructose, "a metabolic effect rather than just a caloric effect".

\section{Katrina Ray}

Original article Lanaspa, M. A. et al. Endogenous fructose
production and metabolism in the liver contributes to
the development of metabolic syndrome. Nat. Commun.
doi:10.1038/ncomms3434

